\title{
La cuestión probatoria de la falta de disposición cuando el Estado ha tenido \\ el propósito de sustraer o blindar al individuo de su responsabilidad por crímenes internacionales
}

Fecha de recepción: 28 de febrero de 2020

Fecha de aprobación: 7 de diciembre de 2020

Doi: https://doi.org/10.12804/revistas.urosario.edu.co/acdi/a.10036

Juan Sebastián Vera Sánchez

Resumen: este trabajo intenta ofrecer una mirada "probatoria" de la falta de disposición cuando el Estado ha tenido el propósito de sustraer o blindar al individuo de su responsabilidad por crímenes internacionales como hipótesis excepcional que permite la admisión del caso para que este sea conocido por la Corte Pena Internacional (CPI) en contrapartida al principio de complementariedad. Se intentará aplicar la visión epistemológica fundaherentista de Susan Haack, con la idea de generar una visión de análisis más objetiva a la hora de aplicar indicadores que tanto la doctrina, como la jurisprudencia han elaborado para saber cuándo un procedimiento judicial no es genuino.

Palabras clave: prueba; falta de disposición; autenticidad.

* Doctor en derecho Universidad de Barcelona. Profesor asistente de Derecho Procesal, Universidad de Chile. Correo electrónico: juansverasanchez@gmail.com Para citar este artículo: Vera Sánchez, Juan Sebastián. "La cuestión probatoria de la falta de disposición cuando el Estado ha tenido el propósito de sustraer o blindar al individuo de su responsabilidad por crímenes internacionales". Anuario Colombiano de Derecho Internacional (ACDI) 14, (2021), 223-256. https:// doi.org/10.12804/revistas.urosario.edu.co/acdi/a.10036 


\title{
The Evidentiary Issue of Unwillingness When the National Decision Was Made for the Purpose of Shielding the Person from International Criminal Responsibility
}

\begin{abstract}
This paper tries to offer an "evidentiary" perspective of "unwillingness" when a State has had the purpose of shielding a person from his/her international crimes responsibility, as an exceptional hypothesis that allows the admissibility of the case by the International Criminal Court (ICC), in the rectification of the complementary principle. An attempt will be made to apply Susan Haack's fundaherentist epistemological theory to generate an objective analysis for applying indicators that scholars and jurisprudence have developed to know when a judicial procedure is not genuine.
\end{abstract}

Keywords: evidence; unwillingness; genuineness.

A questão probatória da falta de disposição quando o Estado tem por objetivo afastar ou isentar o indivíduo de sua responsabilidade por crimes internacionais

Resumo: este trabalho procura oferecer uma visão "probatória" da falta de disposição quando o Estado teve por objetivo afastar ou isentar o indivíduo de sua responsabilidade por crimes internacionais, como hipótese excepcional que permite a admissão do caso para conhecimento pela CPI em contrapartida ao princípio da complementaridade. Será feita uma tentativa de aplicar a visão epistemológica funderentista de Susan Haack. Isso, com a ideia de gerar uma visão de análise mais objetiva ao aplicar indicadores que tanto a doutrina quanto a jurisprudência desenvolveram para saber quando um procedimento judicial não é genuíno.

Palavras-chave: prova; falta de disposição; genuinidade. 


\section{Introducción}

El reconocimiento de la inmutabilidad de la decisión final de los tribunales, en el ejercicio de la jurisdicción, es uno de los pilares fundamentales que contribuyen al sostenimiento del Estado de derecho. El instrumento que se utiliza para ello es la atribución del efecto de cosa juzgada a las adjudicaciones (sentencias definitivas), como una forma de favorecer la estabilidad de las expectativas normativas de los ciudadanos, a través del ejercicio de la jurisdicción, en cuanto atributo de la soberanía de los Estados. Lo anterior redunda en la necesidad de evitar exponer a los ciudadanos a los efectos perniciosos de un segundo procedimiento penal sobre hechos que ya han sido juzgados (ne bis in idem procesal), como una forma de evitar el desgaste propio y el estado de ansiedad que puede sufrir el enjuiciado por efecto de la repersecución sobre los mismos hechos.

El principio general de res judicata es considerado en el ámbito del derecho internacional como uno de esos principios generales del derecho, reconocido por las naciones civilizadas (artículo 38 del Estatuto de la Corte Internacional de Justicia [CI]]). ${ }^{1}$ En este sentido, la decisión jurisdiccional final generaría un efecto sociológico erga omnes o una ficción de verdad, ${ }^{2}$ entre otras, por la necesidad de conseguir seguridad jurídica a través de la evitación de la incoherencia de juicio. ${ }^{3}$

Cosa juzgada y ne bis in idem no solo son instituciones procesales elevadas a principios generales del derecho, sino que también se hallan estrechamente vinculadas con el núcleo de ciertos derechos fundamentales de corte procesal. Por ello, no ha de extrañar que ambos institutos se encuentren previstos en el Estatuto de la Corte Penal Internacional (CPI) o Estatuto de Roma - ER - (artículos 17.2 y 20.3). Sin embargo, el ejercicio de la jurisdicción por parte de diversos tribunales internacionales ha puesto de manifiesto que, muchas veces, el ius dicere de los Estados es utilizado de forma fraudulenta para blindar a los sujetos de

\footnotetext{
1 Rosa Theofanis, "The doctrine of res judicata in international criminal law", International Criminal Law Review, no. 3 (2003): 195.

2 Friedrich Von Savigny, System des heutigen Römischen Rechts. Sechster Band (Berlin: Bei Veit und Comp., 1847), 261; Jordi Nieva, La cosa juzgada (Barcelona: Atelier, 2006), 41-ss.

3 Nieva, La cosa jurgada, 87.
} 
su responsabilidad por crímenes internacionales, especialmente considerando la vigencia del principio de complementariedad. Por ello se habilita la competencia de la CPI, aun cuando los casos hayan sido ya juzgados por tribunales nacionales.

Ahora, el ejercicio de esta facultad de la CPI no ha sido para nada pacífica. Entre otras razones, y dejando al margen las consideraciones políticas, porque implica desconocer el efecto de cosa juzgada de decisiones finales (en el plano interno procesal) en el contexto de la soberanía de los Estados. De otro lado, se opone a la intención del enfoque general del ne bis in idem que pone a salvo el derecho de las jurisdicciones nacionales a tener prioridad en el juicio sobre hechos que originan responsabilidad internacional (en el plano externo). ${ }^{4}$ En otro sentido, la jurisdicción de la CPI, en los casos de falta de disposición de someter al individuo a la justicia, implica un juicio de valor acerca de un sistema de justicia penal doméstico ${ }^{5}$ en relación con el respeto o no de normas fundamentales de justicia. ${ }^{6}$ Ello puede llegar a hacer mutar la función originaria de la CPI, transformándola en un metatribunal, cuestión tampoco deseable. Así, todo lo anterior debe contextualizarse en la compleja tensión de los intereses en juego en el ámbito de la justicia penal internacional. ${ }^{7}$ En efecto, la naturaleza compleja de la CPI —en tanto organización internacional y tribunal de justicia- ${ }^{8}$ debe articular su actuar con el respeto de estos institutos procesales y el principio de complementariedad, de forma de encontrar un punto adecuado y justificado entre el reconocimiento de una acción jurisdiccional soberana de los Estado y la tutela de intereses

4 Mohamed El Zeidy, The principle of complementarity in International Criminal Law (Leiden-Boston: Martinus Nijhoff publisher, 2008), 171.

5 Johan D. van der Vyver, "Personal and Territorial Jurisdiction of the International Criminal Court,” Emory International Law Review 14, no. 1 (Spring 2000): 3.

6 Mark Sternberg. "A Comparison of the Yugoslavian and Rwandan War Crimes Tribunals: Universal Jurisdiction and the Elementary Dictates of Humanity", Brooklyn Journal of International Law 22 (1996): 111-ss.

Lorraine Finlay, "Does the International Criminal Court Protect against Double Jeopardy: An Analysis of Article 20 of the Rome Statute," UC Davis Journal of International Law \& Policy, 15, no. 2 (Spring 2009): 248.

8 Carmen Quesada, "Levantando el velo de la lucha contra la impunidad: la corte penal internacional y sus intereses para actuar", en Actores internacionales contemporáneos, eds. Mónica Rocha, Carmen Quesada y Mario Iván Ureña (Valencia: Tirant lo Blanch, 2019), 225. 
internacionales en los casos en que dichos juzgamientos no son genuinos porque se han efectuado con la finalidad de blindar a los sujetos responsables. En este sentido, el recurso a la soberanía no puede ser un argumento enmascarado que permita seguir tolerando comportamientos deleznables desde el plano de los crímenes internacionales. ${ }^{9}$

La competencia de la CPI es complementaria respecto de los sistemas judiciales nacionales, lo que implica que los Estados tienen la obligación primaria de perseguir los crímenes internacionales. Solo si los Estados demuestran una falta de disposición (unwillingness) o una falta de capacidad (inability) para juzgar a los criminales, entonces la CPI podría llegar a conocer del caso. ${ }^{10}$ En principio, la complementariedad denota un rol secundario de la CPI en relación con el juzgamiento de los hechos por parte de los tribunales de los Estados. ${ }^{11}$ Sin embargo, lo anterior no obsta para reconocer el lugar de privilegio que detenta la CPI en la sanción y juzgamiento respecto de la comisión de crímenes internacionales.

La complementariedad es el mecanismo jurídico mediante el cual se plasma la interacción de la CPI con las jurisdicciones nacionales, que se concentra, entre otras manifestaciones, en una compleja figura de cuestiones de admisibilidad. ${ }^{12}$ El principio se consagra en los artículos 1 y 17 del ER, en virtud del cual la CPI solo ejercerá su jurisdicción

9 Patricia A. McKeon, "An International Criminal Court: Balancing the Principle of Soveriegnty against the Demands for International Justice," St. John's Journal of Legal Commentary 12, no. 2 (Spring 1997): 535-564.

10 Chang-Ho Chung, "The international Criminal Court 20 years after Rome-Achievements and deficits", en The International Criminal Court in turbulent times, eds. por Gerhard Werle y Andreas Zimmermann (The Hague: Asser Press, 2019), 17.

11 "Within the meaning of ICC usage, 'complementarity' denotes a secondary role not in importance but in the sequence of events. In other words, national courts have the first right and obligation to prosecute perpetrators of international crimes, and because ICC jurisdiction is complementary to national courts, ICC jurisdiction can only be invoked if the national court is unwilling or unable to prosecute". Van der Vyver, "Personal and Territorial Jurisdiction", 2000, 66.

En el mismo sentido, Jennifer J. Llewellyn, "A Comment on the Complementary Jurisdiction of the International Criminal Court: Adding Insult to Injury in Transitional Contexts", Dalhousie Law Journal 24, no. 2 (Fall 2001): 193.

12 Alfonso Daza, Elprincipio de complementariedad en el derecho penal internacional (Bogotá: Universidad Católica de Colombia, 2015), 934; El Zeidy, The principle of complementarity, 158.

ACDI, Bogotá, ISSN: 2027-1131/ISSNe: 2145-4493, Vol. 14, pp. 223-256, 2021 
cuando un asunto determinado por el ER - y de cierto umbral de gravedad - no ha sido abordado por las autoridades estatales del país o de los países competentes, o estas no han actuado de acuerdo con los estándares mínimos establecidos en la regulación del ER. ${ }^{13}$ Así, se suele hablar de una complementariedad positiva y negativa, dependiendo si la CPI insta a los Estado a realizar enjuiciamientos genuinos o ejerce su jurisdicción previa constatación de que ellos no se han realizado o se han efectuado de forma manifiestamente defectuosa. Ello se canaliza a través de un juicio de admisibilidad del caso que, de ser positivo, podría llevar ínsita una especie de presunción de que el Estado en cuestión está infringiendo sus obligaciones internacionales. ${ }^{14}$ En efecto, el principio de complementariedad puede ser conceptualizado como una manifestación del fenómeno de jurisdicciones concurrentes, basado en un sistema de soberanía compartida entre la CPI y las jurisdicciones nacionales, lo que constituiría una verdadera última ratio que solo puede activarse frente a la inacción del Estado, su falta de disposición o su incapacidad. ${ }^{15}$ Desde esta perspectiva, el régimen de complementariedad del ER busca alcanzar un equilibrio entre la salvaguarda de la soberanía de los Estados — que se manifiesta en el ejercicio jurisdiccional - y el objetivo de poner fin a la impunidad de los crímenes más atroces. ${ }^{16}$ Si se quiere, se busca con ello administrar la colisión de intereses entre la soberanía del Estado y la necesidad de justicia internacional. ${ }^{17}$

13 Daza, Elprincipio de complementariedad, 94; Claudia Cárdenas, "La cooperación de los Estados con la Corte Penal Internacional a la luz del principio de complementariedad". Revista de Derecho PUCV, Vol. $1 \mathrm{~N}^{\circ} 34$ (1 semester 2010), 284.

14 El mismo autor señala que la complementariedad positiva es el término utilizado por la Oficina de la Fiscalía de la de Corte Penal para describir una serie de estrategias destinada a alentar los procesos nacionales genuinos de enjuiciamiento de los responsables de situaciones que se encuentran bajo en análisis preliminar de la Fiscalía. Daza, Elprincipio de complementariedad..., 95.

15 Héctor Olásolo, "Admisibilidad de situaciones y casos objeto de procesos de justicia de transición ante la corte penal internacional", en Ensayos de Derecho penal y procesal internacional, eds. Jessica Almqvist y Carlos Espósito (Valencia: Tirant lo Blanch, 2011), 14 y 61.

16 Claire Brighton, “Avoiding Unwillingness”: Addressing the Political Pitfalls Inherent in the Complementarity Regime of the International Criminal Court," International Criminal Law Review 12, no. 4 (2012): 630.

17 McKeon, "An International Criminal Court", 1997: 564; Van der Vyver, "Personal and Territorial Jurisdiction", 2000: 3-ss. 
En lo referente a las cuestiones de admisibilidad, el artículo 17 del ER, dispone:

Artículo 17. Cuestiones de admisibilidad

1. La Corte teniendo en cuenta el décimo párrafo del preámbulo y el artículo 1, resolverá la inadmisibilidad de un asunto cuando:

a) El asunto sea objeto de una investigación o enjuiciamiento por un Estado que tenga jurisdicción sobre él salvo que este no esté dispuesto a llevar a cabo la investigación o el enjuiciamiento o no pueda realmente bacerlo;

2. A fin de determinar si hay o no disposición a actuar en un asunto determinado, la Corte examinará, teniendo en cuenta los principios de un proceso con las debidas garantías reconocidos por el derecho internacional, si se da una o varias de las siguientes circunstancias, según el caso:

a) Que el juicio ya haya estado o esté en marcha o que la decisión nacional haya sido adoptada con el propósito de sustraer a la persona de que se trate de su responsabilidad penal por crímenes de la competencia de la Corte, según lo dispuesto en el artículo 5 (...) (negrilla fuera del original)

Por su parte, en materia de ne bis in idem procesal (cosa juzgada) el artículo 20 dispone:

\section{Artículo 20 Cosa jurgada}

1. Salvo que en el presente Estatuto se disponga otra cosa, nadie será procesado por la Corte en razón de conductas constitutivas de crimenes por los cuales ya hubiere sido condenado o absuelto por la Corte.

2. Nadie será procesado por otro tribunal en razón de uno de los crímenes mencionados en el artículo 5 por el cual la Corte ya le bubiere condenado o absuelto.

3. La Corte no procesará a nadie que haya sido procesado por otro tribunal en razón de hechos también probibidos en virtud de los artículos 6, 7 u 8 a menos que el proceso en el otro tribunal: 
a) Obedeciera al propósito de sustraer al acusado de su responsabilidad penal por crímenes de la competencia de la Corte; 0

b) No bubiere sido instruido en forma independiente o imparcial de conformidad con las debidas garantías procesales reconocidas por el derecho internacional o lo bubiere sido de alguna manera que, en las circunstancias del caso, fuere incompatible con la intención de someter a la persona a la acción de la justicia (negrilla fuera del original).

La doctrina ha reconocido que los tres criterios para evaluar la falta de disposición presentan una vaguedad que dificulta su aplicación. ${ }^{18}$ Por su parte, el "propósito de sustraer al acusado de su responsabilidad por los crímenes de competencia de la Corte" se ha considerado el núcleo del criterio de la "falta de disposición", 19 cuyo contexto nomogenético se explica teniendo en cuenta la situación de Yugoslavia, donde los tribunales tenían la capacidad de juzgar a los autores de crímenes internaciones, ${ }^{20}$ pero se percibía que políticamente no existía la voluntad de llevar procesos judiciales genuinos en contra de ellos. ${ }^{21}$ Ahora, la falta de disposición presupone una decisión consciente: el Estado debe estar decidido a no enjuiciar de forma genuina; de lo contrario, no hay problema de disposición. ${ }^{22}$ En efecto, el artículo 17.2 a) del ER contiene

18 Vincent Dalpe, "On the Difficult Case for a Functional Interpretation of the Unwillingness Criterion before the International Criminal Court," Journal of International Law and International Relations 13, no. 2 (Fall 2017): 50; Mark S. Ellis, "The International Criminal Court and Its Implication for Domestic Law and National Capacity Building," Florida Journal of International Law 15, no. 2 (Fall 2002): 237, entre otros.

19 Jo Stigen. The relationship between the international criminal court and national jurisdictions (Leiden, Boston: Martinus Nijhoff Publisher, 2008), 251.

20 Véase, ICTY, Prosecutor v. Duško Tadić, Case No. IT-94-1-AR72, Decision on the Defence Motion for Interlocutory Appeal on Jurisdiction, 2/10/1995, para. 58.

21 Brighton, "Avoiding Unwillingness", 2012: 640; Llewellyn, “A Comment on the Complementary", 194. Según El Zeidy, uno de los primeros precedentes de juicios no genuinos se puede encontrar en los "Juicos de Leipzig" frente al Reichsgericht. El Zeidy, The principle of complementarity..., 2008: 163-ss.

22 Stigen, The relationship between the international criminal court, 2008: 251. 
un test para discernir acerca de la mala fe del Estado, verificando la efectividad y autenticidad del procedimiento judicial del orden nacional. ${ }^{23}$

De otro lado, con esta atribución de la CPI se busca que los procesos judiciales respeten los mínimos del debido proceso, así como la imparcialidad e independencia del juzgado (artículo 20.3 b) ER). Sin embargo, la vaguedad ínsita a la noción "debido proceso" impide que esta presente una función de limitación estable, concreta y con elementos definidos respecto de los juzgamientos domésticos. Iguales problemas de vaguedad se generan cuando se utiliza la noción de "estándares internacionales" como límite. ${ }^{24}$

El reproche respecto de enjuiciamientos no genuinos no es algo exclusivo de las atribuciones de la CPI. Por ejemplo, la Corte Interamericana de Derechos Humanos (Corte IDH) ha acuñado el término "cosa juzgada fraudulenta" para referirse al efecto que produce este mismo tipo de procedimientos judiciales no genuinos, a partir de los cuales no tiene aplicación el principio de ne bis in ídem procesal, pese a existir un procedimiento judicial ya finalizado sobre los mismos hechos. En efecto, la Corte IDH ha señaló en el Caso Almonacid Arellano y otros c. Chile:

para la Corte, si bien el ne bis in ídem es un derecho bumano reconocido en la CADH, no es un derecho absoluto. Asi, se vería vencido por las siguientes circunstancias:

1) Cuando la actuación del tribunal que conoció del caso actuó con el propósito de sustraer al responsable de una violación de los DDHH de su responsabilidad penal

2) Faltaron las garantías procesales de imparcialidad o independencia

3) No bubo "intención real de someter al responsable a la acción de la justicia" (parr.154) (negrilla fuera del original). ${ }^{25}$

\footnotetext{
23 Williams Schabas y Mohamed El Zeidy, "Art.17", en The Rome statute of The International Criminal Court, eds. Otto Triffterer y Kai Ambos (Berlin: Beck/Hart, 2016): 819. 24 Ellis, “The International Criminal Court...", 2002: 226.

25 Este criterio también ha sido ratificado por la CIDH en el caso "Nadege Dorzema y otros vs. República Dominicana” (parr. 195), "Caso Carpio Nicolle y otros vs. Guatemala" (párr. 131).
} 
Igual reproche normativo puede encontrase en artículo 20 del Estatuto del Tribunal Internacional para Ruanda (1994) y en los artículos 9 y 10 (2) b) del Estatuto del Tribunal Internacional para la Antigua Yugoslavia (1993).

Por otro lado, el alcance y límites del trabajo de la CPI están determinados por los Estados parte, por lo que el rol que esta pueda tener en la lucha contra la impunidad de los crímenes internacionales más graves depende, en buena medida, de la confianza que le otorguen los Estados. ${ }^{26}$ Esta confianza se puede poner en riesgo cuando el ejercicio de atribuciones excepcionales de la $\mathrm{CPI}^{27}$ — como lo es entrar a conocer un caso ya juzgado pese al principio de complementariedad- no puede ser reconstruido consistentemente a partir de un orden y sistematización de los casos e indicios que se han utilizado para aplicar la norma de los artículos 17.2. a) y 20.3 del ER. La evidente cuestión política que subyace a la aplicación del principio de complementariedad en el cual se insertan la aplicación de estos artículos, quizás, ha impedido a la doctrina dedicar buena parte de su trabajo a depurar el tratamiento de los indicadores de juicios no genuinos. En efecto, la literatura es abundante respecto del principio de complementariedad, pero escasa respecto de esta importante previsión normativa. ${ }^{28}$

De otro lado, debemos considerar también que las acciones de la CPI precisan de cooperación de los Estados (p. ej., en materia de prueba y órdenes de detención). ${ }^{29}$ La importancia de esta cooperación constituye una característica distintiva de los procedimientos penales internacionales. ${ }^{30}$ Sin embargo, parece poco conveniente entrar a conocer un caso calificando, de entrada, como fraudulenta la acción de los tribunales/

\footnotetext{
26 Gerhard Werle y Andreas Zimmermann, "Introduction”, en The International Criminal Court in turbulent times, eds Gerhard Werle y Andreas Zimmermann (The Hague: TMC Asser Press, 2019), 2.

27 Linda Carter, "The Principle of Complementarity and the International Criminal Court: The Role of Ne Bis in Idem," Santa Clara Journal of International Law, 8, no. 1 (2010): 194.

28 Dalpe,"On the Difficult Case for a Functional Interpretation...", 2017: 51.

29 Quesada, “Levantando el velo de la lucha contra la impunidad...”, 1997: 237.

30 Claus Kress, "The Procedural Law of the International Criminal Court in Outline: Anatomy of a Unique Compromise," Journal of International Criminal Justice, 1, no. 3 (December 2003): 614. En el mismo sentido, Ellis,"The International Criminal Court...", 2002: 219-ss.
} 
Estado respecto de los cuales se precisará de cooperación para el juzgamiento. Por lo demás, el poder institucional de la CPI se basa en la anuencia de los Estados, ${ }^{31}$ por lo que todo ello hace más compleja aún la declaración de admisibilidad de estos casos.

\section{Tesis epistemológica de la cuestión}

La cuestión acerca de si la actuación del Estado demuestra o no una falta de disposición que se traduzca en el propósito de sustraer a la persona de su responsabilidad internacional por los crímenes cometidos se ha abordado principalmente desde una esfera valorativo-normativa, por un lado, y a partir de la conjunción de ciertos criterios individuales que manifiestan dicho propósito (indicadores), por otro. Mi intención es plantear la cuestión desde una arista epistemológica, — si se quiere, de prueba-, poniendo el énfasis en que dicha cuestión también plantea una duda "epistémico-fáctica" acerca de si el Estado en cuestión se encuentra o no en dicha situación. Es decir, si en los "hechos" esta cuestión acaece o no. En efecto, los indicadores que permitirían inferir la intención de blindar a un sujeto de su responsabilidad dependen en gran medida de las circunstancias fácticas de cada caso particular. ${ }^{32} \mathrm{La}$ descomposición de la cuestión de la falta de disposición en un análisis fáctico se aviene, además, con la intención de reducir el "subjetivismo" de la valoración de falta de disposición en favor de una cierta mirada objetiva que proporciona la valoración racional de los antecedentes fácticos y el entendimiento, por ejemplo, de la autenticidad (genuineness) del procedimiento como algo a ser valorado de forma objetiva. ${ }^{33}$

31 Carter, "The Principle of Complementarity...", 2010: 194; Brighton, “Avoiding Unwillingness...", 2012: 632.

32 El Zeidy, The principle of complementarity, 2008: 166; Schabas y El Zeidy, “Art.17”, 2016: 819; Van der Vyver, "Personal and Territorial Jurisdiction...”, 2000: 74. Así también en Report of the Preparatory Committee on the Establishment of an International Criminal Court, Vol I, U.N. GAOR, $51^{\circ}$ sesión., Supp. No. 22, at 30-41, par.161, U.N. Doc. A/51/22 (1996)".

33 El Zeidy, The principle of complementarity..., 2008: 163 y 168; Ellis, “The International Criminal Court...", 2002: 237. La version en inglés del Estatuto de Roma dispone en su artículo 17 a): "The case is being investigated or prosecuted by a State which has jurisdiction over it, unless the State is unwilling or unable genuinely to carry out the investigation or prosecution." Sin embargo, el adjetivo "genuino" no tiene un reflejo literal expreso en la versión castellana del ER (citado supra), perdiéndose con ello la 
Sin embargo, la cuestión epistémica no es excluyente de la cuestión normativa. Los antecedentes probatorios sirven para corroborar un enunciado fáctico que puede dar cuenta de la situación de falta de disposición de someter a la persona a la responsabilidad internacional, ya sea a partir de un juicio de subsunción en sentido estricto, ya sea a partir de un juicio valorativo-normativo de la situación. Lo que quiero decir es que es también posible analizar la falta de disposición considerando que los enjuiciamientos no genuinos se construyen sobre la base de hechos o enunciados fácticos que, probados, permiten inferir o adscribir que los Estados se encuentran dentro del margen de dicha previsión normativa.

\section{Tesis de Haack}

Cómo podemos conocer una realidad desconocida, con una justificación intersubjetivamente compartida, es una cuestión de la que ya se han ocupado filósofos de la ciencia, filósofos del derecho y probatoristas, entre otros. Es el cuestionamiento que intenta responder, en el ámbito probatorio, al interior de los procedimientos judiciales a la finalidad institucional de la búsqueda de la verdad y el concepto de probabilidad lógica, ${ }^{34}$ entre otros, como términos aceptados por las teorías racionalista de la prueba. En particular, y para los efectos de este trabajo, seguiremos la tesis epistémica de Haack basada en una combinación entre el fundacionalismo y el coherentismo (tesis fundaherentista). La teoría de Haack sobre la verdad no solo se intenta aplicar a los requerimientos y teorías de las ciencias en sentido estricto, sino también a otros tipos de proposiciones de otros campos. ${ }^{35}$ Warrant es una categoría epistemológica, cuya presencia se extrae a partir del quehacer científico, aunque no exclusiva de dicho campo. ${ }^{36}$ Según Taruffo, el término warrant en este contexto puede ser traducido como confirmación. Haack considera importante

riqueza de la discusión que para la materia aporta dicho vocablo en relación con la falta de disposición o de capacidad.

34 Jordi Ferrer, La valoración racional de la prueba (Barcelona: Marcial Pons, 2007): 62-ss; William TwINING, Rethinking evidence, $2^{\text {nd }}$ editions (Cambridge: University Press. Cambridge, 2006): 35-ss.

35 Susan Haack. "Of Truth, in Science and in Law". Brooklyn Law Review, Vol. 73 N³, (2008): 993.

36 Susan Haack. "Defending science-within reason". Principia, 2 (3), (1999): 199; Susan Haack, "Federal Philosophy of sciencie: A deconstruction and a reconstruction". 
la distinción entre descubrimiento y justificación, que no es más que la distinción entre estándares de buena evidencia (standars of good evidence) y reglas o principios para conducir una investigación. ${ }^{37}$ Ello significa que la confirmación de la afirmación depende de qué tan buena sea la evidencia respecto de ese alegato (claim).$^{38} \mathrm{La}$ forma de cómo se confirma empíricamente una afirmación depende de que tan bien sea apoyada por la experiencia y antecedentes creíbles; de cómo se garantiza la credibilidad de los antecedentes (independientes de la afirmación en cuestión), y de cuánta evidencia relevante aporta la evidencia que se tiene a la vista. ${ }^{39}$ Cada prueba que se agrega a otra aumenta el grado de confirmación de la hipótesis. ${ }^{40}$ En este sentido, la confirmación de la hipótesis es una cuestión gradual (en función de las pruebas que se refieran a ella en un determinado momento o dependen del grado de apoyo [supportiveness] que tenga respecto de la hipótesis). ${ }^{41}$

En lo particular, considero que se da valor a la vinculación probatoria entre las distintas hipótesis cuando se considera que la evidencia es ramificada, al modo de un crucigrama. Desde esta perspectiva, la razonabilidad de la palabra que se introduce dependerá de lo bien que encaje con las ideas y entradas ya completadas. ${ }^{42}$ Ello se traduce en el primer requisito para medir el grado de confirmación de una afirmación: "how strong the connection is between the evidence and the conclusión" (supportivenes), ${ }^{43}$ a los cuales agrega, posteriormente, lo que ella

New York University Journal of Law and Liberty, N²2, vol. 5, (2010): 394 ss; Haack, "Of Truth", 998.

37 Michele Taruffo, Simplemente la verdad: el juez y la construcción de los hechos. Traducc. Daniela Accatino Scagliotti (Madrid-Barcelona: Marcial Pons, 2010), 236.

38 Haack, “Of Truth...”, 2008: 997; Haack, “Defending science...”, 1999: 195.

39 Haack, "Defending science...", 1999: 198.

40 Taruffo, Simplemente la verdad..., 2010: 236.

41 Susan Haack, "Proving causation: The holism of Warrant and the Atomism of Daubert". Journal of Health and Biomedical Law, No 4, (2008): 263.

42 Haack, "Proving causation...", 2008: 263.

43 La autora lo explica (Ibid), de la siguiente manera: "How supportive evidence is of a conclusion depends, to put it quite briefly and roughly, on how well the evidence and the conclusion fit together to form an explanatory account". Ibíd. 
denomina independent security ${ }^{44}$ y comprehensivenes. ${ }^{45}$ Estos tres elementos para la autora son los determinantes de la calidad probatoria. Por ejemplo, la combinación de la evidencia también puede mejorar el requisito de la independent security, del mismo modo que completar una entrada del crucigrama que calce con otra nos hace pensar que la palabra introducida es correcta. ${ }^{46}$ Igualmente, si una palabra del crucigrama calza con otra, y así sucesivamente, aumenta la posibilidad de que el todo sea correcto, que si evaluamos una sola palabra. Según Taruffo, todo esto se traduce en que la afirmación no resulte excluida, falsificada o contradicha por hipótesis alternativas referidas al mismo hecho. ${ }^{47}$

En otras palabras, cuando queramos conocer una situación fáctica desconocida, los antecedentes probatorios no deben ser solo analizados en términos lineales e individuales respecto de la realidad a comprobar (atomismo), sino también en términos de coherencia o de valoración conjunta u holista. Los antecedentes también pueden "imprimir" una imagen de conjunto del suceso fáctico dubitado, cuestión no excluyente sino más bien de concurrencia conjuntiva con la visión individual. Algo de ello hay en algunos pronunciamientos de la Corte IDH, cuando se señala que las declaraciones rendidas por las presuntas víctimas no pueden ser valoradas aisladamente sino dentro del conjunto de las pruebas del proceso - Caso de la "Panel Blanca" (Paniagua Morales y otros); Caso Chitay Nech y otros; Caso Manuel Cepeda Vargas_-, lo que evoca una cierta idea de corroboración de la declaración, de una valoración global u holista.

Esta es, en versión de Haack, la mejor alternativa epistémica para acercarnos con el menor margen de error posible al conocimiento de una realidad desconocida como puede ser, en nuestro caso, la falta de disposición del Estado con el propósito de sustraer a un sujeto de la responsabilidad internacional. En efecto, la cuestión jurídica termina por eliminar casi por completo la cuestión fáctica de la situación de "blindaje" y, cuando está presente, los antecedentes son valorados de una forma atomista o individual, dejando de considerar la perspectiva de coherencia o de conjunto. La cuestión se ve claramente perjudicada

\footnotetext{
44 Ello significa: "how solid the evidence itself is, independent of the conclusion".

45 Ello significa: "how much of the relevant evidence the evidence includes".

46 Haack, "Proving causation...", 2008: 271.

47 Taruffo, Simplemente la verdad..., 2010: 236.
} 
por tratarse de un juicio de admisibilidad y no de prueba rendida en la fase de juicio con todos los límites y seguridades que ello implica para los intervinientes.

Por ejemplo, algunos autores han considerado que frente a la vaguedad y falta de precisión de los criterios de falta de disposición (artículos 17.2 a y 20.3 a, ER), la CPI ha tenido que crear o inventarse indicadores, muchas veces extraídos de la jurisprudencia de otros tribunales $^{48}$ o de posiciones doctrinales aisladas, dejando la puerta abierta para que los Estados orquesten ensayos falsos de enjuiciamientos. ${ }^{49} \mathrm{De}$ hecho, a mi modo de ver, esta imprecisión sistemática y epistémica se sigue en el Documento de politica general sobre exámenes preliminares de la Oficina de la Fiscalia de la Corte penal Internacional de 2013, cuyo párrafo 51 señala:

51. La intención de sustraer a una persona de su responsabilidad penal puede evaluarse a la luz de indicadores tales como los siguientes: medidas manifiestamente insuficientes durante la investigación o el enjuiciamiento; desviaciones de las prácticas y procedimientos establecidos; pasar por alto pruebas o asignarles un peso insuficiente; intimidación de víctimas, de testigos o de personal judicial; incongruencia entre decisiones frente y la prueba presentada; insuficiencias manifiestas en la formulación de cargos y los modos de responsabilidad en relación con la gravedad de la conducta alegada y el rol atribuido al acusado; conclusiones judiciales equivocadas a causa de una identificación errónea, un examen forense defectuoso, fallas en la divulgación, pruebas fabricadas, declaraciones manipuladas o extraidas mediante coacción, o decisiones indebidas de admisión o no admisión de pruebas; falta de asignación de recursos al procedimiento de que se trata en comparación con la capacidad global, y negativa a dar información a la CPI o a cooperar con ella. ${ }^{50}$

Como se ve, se trata de "indicadores" que en su formulación evocan una enunciación conjunta de factores aislados o individuales, sin que se haga una mención de la perspectiva holista o de conjunto,

48 El Zeidy, The principle of complementarity, 2008: 163-ss.; Schabas y El Zeidy, "Art.17”, 2016: 819; Dalpe, “On the Difficult Case...", 2017: 53.

49 Dalpe, “On the Difficult Case...", 2017: 53.

50 Corte Penal Internacional, Oficina de la Fiscalía, Documento de política general sobre exámenes preliminares, 14, https://www.icc-cpi.int/iccdocs/otp/OTP-PolicyPaper-on-Preliminary-Examinations-November2013-SPA.pdf 
que impriman en la imagen global una probable situación de falta de disposición del Estado. La cuestión es de relevancia porque la doctrina ha reconocido, a partir de toda la teorización que se ha hecho respecto del principio de complementariedad, que los Estados tienen un cierto margen de maniobra para el ejercicio de la jurisdicción como atributo de su soberanía — lo cual podría incluir algunas acciones aisladas negligentes- sin que ello signifique necesariamente una falta de disposición de someter al individuo a la justicia, entre otras razones, por el carácter de ultima ratio de la jurisdicción de la CPI para enjuiciar los crímenes internacionales. De otro lado, muchas veces la comprensión y aplicación de estos indicadores no es del todo acabada. En el caso Katanga, por ejemplo, se hizo referencia a la falta de disposición sin señalar ninguno de los tres criterios del artículo 17.2 del ER. ${ }^{51}$

\section{La tesis epistemológica "mixta" y algunos elementos normativos presentes en el ER}

Hay varios elementos en el ER que aconsejan tener presente no solo la valoración individual de los antecedentes, sino también una imagen global de la situación de falta de disposición originada en ejercicios judiciales no genuinos. En efecto, desde el plano orgánico, la falta de disposición es un atributo normativo predicable del Estado, ${ }^{52}$ pero no de una persona en particular. Por supuesto, los órganos del Estado actúan principalmente sobre la base de directrices elaboradas por quienes los encabezan, pero no deben dar cuenta de un acto aislado, sino de una "intención o propósito"- con claro sentido literal "subjetivo" lo cual ha provocado no pocos problemas para el tema que se trata $-{ }^{53}$ que se podría traducir, en términos organizacionales, en que el ejercicio jurisdiccional simulado o falaz sea la expresión de un "estado" o "situación organizacional" de impunidad en favor de un determinado sujeto.

Desde el plano probatorio, y dejando claro que las organizaciones en cuanto personas jurídicas no manifiestan cuestiones volitivas o al menos no equiparables con las personas naturales o físicas, la "intención"

\footnotetext{
51 Dalpe, “On the Difficult Case...", 2017: 56.

52 Stigen, The relationship between the international criminal court..., 2008: 253.

53 Stigen, The relationship between the international criminal court..., 2008: 259; El Zeidy, The principle of complementarity..., 2008: 168.
} 
o "propósito" muy pocas veces presentará prueba directa ${ }^{54}$ por lo que deberá reconstruirse principalmente sobre la base de prueba indirecta, circunstancial o por indicios, lo cual nuevamente evoca la necesidad de abordar también la cuestión desde un plano holístico o global y no solo a partir de una reunión de indicadores.

Desde el plano del diseño normativo del ER, la prerrogativa de declarar admisible los casos pese a que haya un ejercicio jurisdiccional doméstico en la situación de "blindaje", es una excepción a la inadmisibilidad propia de los casos que han sido juzgados en el ámbito interno de manera "genuina". Con ello también se genera un régimen excepcional respecto de las atribuciones jurisdiccionales complementarias de la CPI. Así, la competencia de la CPI frente a decisiones jurisdiccionales falaces o simuladas no debiera ser — bajo ningún punto — una medida de "primera línea" frente a procedimientos domésticos irregulares, exigiéndose de ello una cuidadosa aplicación. Como ha reconocido la doctrina: ningún Estado que ratificó el ER hubiese esperado en ese momento que se utilizara la compentencia de la CPI como una "tercera instancia" o "instancia revisora" de lo actuado por tribunales nacionales, ${ }^{55}$ especialmente cuando dicha decisión — de entrada - es unilateral. ${ }^{56}$ John T. Holmes ha señalado que la premisa subyacente al régimen de complementariedad era asegurar que la CPI no interferiría con investigaciones o persecuciones nacionales, excepto en los casos más obvios. ${ }^{57}$ Ello dista de la posición adoptada por la CPI en el caso Lubanga, donde no pareció mostrar mucha deferencia hacia las jurisdicciones nacionales. ${ }^{58}$ Muchas veces, la complementariedad parece ceder frente a intereses institucionales más fuertes de la CPI, ${ }^{59}$ como también parece haber quedado de manifiesto en el caso Katanga-Ngudjolo Chui, donde se desechó en sede de

54 Stigen, The relationship between the international criminal court..., 2008: 259.

55 Brighton, “Avoiding Unwillingness...", 2012: 640.

56 Ellis, “The International Criminal Court..”, 2002: 240.

57 John T. Holmes. "Complementarity: National vs. The ICC”, in The Rome statute of the International Criminal Court, eds. Antonio Cassese, Paola Gaeta y John W. R. D. Jones (Oxford: Oxford University Press, 2002), 674.

58 Ximena Fuentes, "El principio de complementariedad en la práctica de la Corte Penal Internacional”, Estudios Internacionales, 169, (2011): 134.

59 Quesada, “Levantando el velo de la lucha...", 2019: 237. 
admisbilidad el ne bis in idem alegado pese a que el acusado ya había sido juzgado por tribunales congoleños. ${ }^{60}$

No debe olvidarse, por otro lado, que las normas de los artículos 17.3 a) y 20.3 del ER son unas reglas que vienen a resolver un "conflicto de jurisdicciones", ${ }^{61}$ en este caso específico y excepcional en favor de la competencia de la CPI.

Todo lo anterior genera una "carga" argumentativa o justificatoria para la CPI si quiere entrar a conocer estos casos basada en esta prerrogativa, ${ }^{62}$ entre otras razones, porque ella también debe conjugarse con el sentido y alcance del principio de complementariedad. A mi modo de ver, ello no exige elevar el estándar de prueba de la falta de disposición que, en este caso, seguirá siendo el de la probabilidad prevaleciente, ${ }^{63}$ sino simplemente reforzar la argumentación o justificación de esta atribución, por ejemplo, integrando la perspectiva holista o de coherencia de la valoración de los antecedentes que habiliten la admisión del caso.

\section{Algunos Indicadores del propósito de sustraer a una persona de la responsabilidad por crímenes internacionales}

Creo conveniente presentar los indicadores más importantes para revisar su fundamentación, siguiendo para ello la exposición que hace Stigen. ${ }^{64}$ En todo caso, la multiplicidad y amplitud de criterios será una problemática que se debe aceptar y con la que se debe trabajar, pues no es posible prever de forma exhaustiva todos los escenarios que reflejen la intención de blindar a un sujeto de su responsabilidad por los crímenes cometidos. ${ }^{65}$

60 Véase, Prosecutor v. Germain Katanga and Mathieu Ngudjolo Chui, Case $\mathrm{N}^{\circ}$. ICC-01/04-01/07, Decision on the Confirmation of the Charges, at 209-12 (Sept. 30, 2008). También, en Finlay, "Does the International Criminal Court", 248.

${ }^{61}$ Brighton, "Avoiding Unwillingness...", 2012: 636; Stigen, The relationship between the international criminal court..., 2008, 232.

62 Llewellyn, “A Comment on the Complementary...”, 2001: 202.

63 Stigen, The relationship between the international criminal court..., 2008, 178.

64 Stigen, The relationship between the international criminal court..., 2008, 265-ss.

65 Schabas y El Zeidy, “Art.17”, 2016, 819. 


\subsection{Renuencia a cooperar con la Fiscalía de la CPI}

Como afirmaba, el éxito del funcionamiento de la CPI depende en gran medida de la colaboración de los Estados, cuestión sabida por los miembros de la comunidad internacional. Si un Estado es reacio a cooperar con el Fiscal de la CPI, esto podría indicar que el Estado busca ocultar algo. El Estado es, por ejemplo, reacio a proporcionar al fiscal información sobre una investigación o enjuiciamiento en curso o finalizado. Si se completa el rechazo, incluso podría justificar una inversión de la carga de la prueba en cuanto a la autenticidad del procedimiento. En este sentido, la simple renuencia en el contexto de operatividad de la CPI no puede considerarse una actitud claramente neutral de un Estado.

Este criterio también es recogido por la Oficina de la Fiscalía de la CPI, donde expresamente señala que es un indicador la negativa a dar información a la CPI o a cooperar con ella.

\subsection{Limitación al acceso de la justicia}

En estos casos se debe evaluar si el Estado ha facilitado el acceso a las autoridades policiales y judiciales para presentar denuncias. El acceso particularmente difícil puede ser indicador de un enjuiciamiento no genuino. Los procedimientos deben estar disponibles y ser completos para todos los que quieran presentar quejas. En efecto, la posibilidad de impetrar acciones judiciales a quienes posean legitimidad activa está incluido en el derecho de tutela judicial efectiva, que se conculca cuando el acceso a tribunales se dificulta.

Otro problema relacionado podría ser la falta de asistencia legal que permita al público abordar la cuestión adecuadamente. Ello, además, debe ser puesto en relación con que, en la mayoría de los sistemas penales acusatorios modernos del ámbito románico continental — con influencia anglosajona-, el ejercicio de la acusación por delitos de acción penal pública corresponde en exclusiva al ministerio público.

\subsection{Inadecuada asignación de recursos}

El hecho de que un Estado no asigne recursos a investigaciones y enjuiciamientos podría ser indicativo de un propósito de proteger a los sujetos de los procedimientos. En efecto, muchas de las medidas de investigación que se concretan en ciertos antecedentes de prueba, que revisten un gasto 
muchas veces difícil de ser asumido por los intervinientes. Una forma de torcer el resultado del juicio es, precisamente, disminuyendo la fluidez financiera que posibilita el acceso a medios óptimos para fundar una imputación en contra de un sujeto que se intenta blindar.

Este criterio también es recogido por la Oficina de la Fiscalía de la CPI, señalando expresamente que es un indicador la falta de asignación de recursos al procedimiento de que se trata en comparación con la capacidad global que se tenga. También este criterio ha tenido reflejo en la doctrina. ${ }^{66}$

\subsection{Acceso y seguridad de los investigadores}

El fiscal de la CPI también debe evaluar si el marco procesal de un Estado otorga a los investigadores acceso irrestricto y seguro a todas las pruebas, incluidos los testigos y la escena del crimen. Por ejemplo, la Comisión de Verdad y Reconciliación de Chile (en el contexto de la dictura militar de Pinochet), que en realidad no era un organismo de investigación criminal, fue criticada masivamente porque no pudo entrevistar a miembros de las fuerzas de seguridad, ${ }^{67}$ en virtud de una prohibición institucional al respecto. Si el Estado proporciona acceso "formal" a los antecedentes de la investigación, sin medidas que aseguren una toma de contexto "material" con las pruebas, investigadores, víctimas y testigos, en definitiva, podrían quedar excluidos total o parcialmemente del enjuiciamiento.

Muchas veces, la actuación de querellantes y de otros legitimados activos no institucionales puede ayudar a la obtención de medios de prueba o a la depuración de las líneas de investigación que se lleven a cabo. Lo peligroso de todo ello es que, con acciones impeditivas del conocimiento de las pruebas o antecedentes por parte del Estado, se puede terminar por condicionar — indirectamente- el resultado final del pleito. De ahí la importancia de cautelar la efectiva participación en el enjuiciamiento de los diversos actores involucrados.

\footnotetext{
66 Wolffhügel, Christian, "La falta de disposición y de capacidad de los sistemas judiciales domésticos, en el marco del principio de complementariedad. A propósito del Estatuto de la Corte Penal Internacional." Civilizar. Ciencias Sociales y Humanas, 9, no. 17 (2009):79-ss.
}

${ }_{67}$ Stigen, The relationship between the international criminal court..., 2008, 268. 


\subsection{Intimidación de los actores del procedimiento}

Otra forma de generar una situación de no autenticidad judicial es intimidando o amenazando a investigadores, jueces o testigos como una forma de torcer o modificar la información que proporcionen en relación con la imputación del sujeto que se intenta blindar. El éxito de la investigación depende de que las víctimas y testigos puedan acercarse a quienes llevan a cabo la investigación sin temores. De lo contrario, testigos o víctimas podrían no comparecer o proporcionar información parcial. ${ }^{68}$

Este criterio también es recogido por la Oficina de la Fiscalía, donde expresamente señala que es un indicador el ejercicio de intimidación de víctimas, de testigos o de personal judicial y la extracción de prueba mediante coacción.

\section{6. Órganos judiciales especiales}

Si los poderes estatales asignan facultades a órganos jurisdiccionales especiales, como las comisiones de investigación militar y los tribunales militares (tribunales especiales), esto podría implicar que la ley ya no se aplica de forma independiente. También este criterio ha tenido reflejo en la doctrina ${ }^{69}$ Los tribunales ad hoc en el orden doméstico, además, se oponen a la garantía del juez natural o de existencia de tribunal imparcial establecido con anterioridad a la comisión de los hechos (p. ej., artículo 8 de la CIDH).

\subsection{Minuciosidad insuficiente en el procedimiento}

En estos casos, se debe evaluar si el procedimiento doméstico ha sido óptimo desde el punto de vista de la suficiente minuciosidad. Igualmente debe tenerse en cuenta que el principio de complementariedad otorga a los Estados un generoso margen de apreciación no solo en cuanto a cómo llevan a cabo la investigación, sino también en cuanto a la exhaustividad..$^{70}$ Desde esta perspectiva, no cualquier falta de minuciosidad puede ser un indicador de un procedimieto no genuino. Lo que

\footnotetext{
68 Ibíd.: 271.

69 Wolffhügel, "La falta de disposición...”, 2009: 86.

70 Stigen, The relationship between the international criminal court..., 2008, 273.
} 
se requiere es que el esfuerzo refleje la intención del Estado de llevar al autor ante la justicia. Sin embargo, una investigación superficial tendrá menos probabilidades de tributar a un enjuiciamiento genuino. ${ }^{71}$ Debe tenerse en cuenta el hecho de que investigar crímenes internacionales es intrínsecamente complejo, porque, en definitiva, se trata de una obligación de medios y no de resultado —así, CIDH, caso "Velasquez con Honduras" -.$^{72}$

En efecto, como ha reconocido la doctrina, cuanto más exhaustiva y seria sea la investigación realizada en el ámbito nacional, más dificil será encontrar prueba de una intención de blindaje y viceversa. ${ }^{73}$ El Tribunal Europeo de Derechos Humanos (TEDH) ha dejado claro que las investigaciones deben ser serias y efectivas y, no cumplíendose dichas condiciones sin adecuadas justificaciones — que hace resurgir la mala fe del Estado-, se construye una presunción de que el Estado ha querido blindar al sujeto de su responsabilidad internacional. ${ }^{74}$ En efecto, el TEDH, en el caso Nachova y otros c. Bulgaria, ${ }^{75}$ da una serie de criterios para estimar que el juzgamiento del Estado fue efectivo: a) las autoridades nacionales debieron haber adoptado todos los pasos disponibles para asegurar la prueba concerniente al incidente, incluyendo testigos y prueba pericial o forense; b) las conclusiones de la investigación deben ser basadas en un análisis objetivo, imparcial de todos los elementos relevantes, y c) cualquier deficiencia en la investigación que perjudica su capacidad para establecer las circunstancias del caso o que el sujeto responsable haya incumplido una medida de efectividad del procedimiento. En el caso Timurtas c. Turquía, el TEDH asimió la falta de efectividad de investigaciones que había sido superficiales. ${ }^{76}$ En el caso Kuznetsov c. Ucrania, el mismo tribunal consideró que la imposibilidad de encontrar registros que pudieran reconstruir paso a paso la naturaleza

71 El Zeidy, The principle of complementarity..., 2008, 175-ss.

72 Velasquez Rodríguez c. Honduras, 29 de julio de 1988, Serie C. N 4, párr 177.

73 El Zeidy, The principle of complementarity..., 2008: 175; Schabas y Zeidy, “Art.17”, 2016: 819 .

74 Schabas y Zeidy, “Art.17”, 2016, 819.

75 Nachova and others v. Bulgaria, Applications Nos. 43577/98 and 43579/98, TEDH, 6 de julio 2005, par. 113.

76 Timurtas vs. Turquía, Application 23531/94, TEDH, Sentencia (Merits and Just Satisfaction), 13 de junio de 2000, pars 88-ss. 
de la investigación llevada a cabo es un factor que revela la mala fe del Estado respecto de llevar a cabo una investigación seria. ${ }^{77}$ En el caso Tepe c. Turquía, la omisión de la prueba pericial en el caso de una muerte no natural, llevando a que el fiscal omitiere la realización de una autopsia, se consideró una omisión "sorprendente", que condujo a considerar que el procedimiento fuera ineficaz. ${ }^{78}$

La efectividad de la investigación, de acuerdo a la jurisprudencia del TEDH, debe ser evaluada de acuerdo con dos "tests". El primero, generalmente aplicable en los casos de violaciones a los artículos 2 y 3 del Convenio Europeo de Derechos Humanos, consiste en estimar que la investigación es apta para conducir a la identificación y castigo de los responables. El segundo, se refiere a la evaluación de las autoridades nacionales en torno a lo que que debieron hacer o no para conseguir los objetivos del primer test. ${ }^{79}$

En el consorcio latinoamericano, la CIDH ha señalado en el caso Velasquez vs. Honduras, que si bien la realización de la investigación en casos penales es una obligación de medios, ella igualmente debe emprenderse con seriedad y "no como una simple formalidad condenada de antemano a ser infructuosa" (parr. 177). ${ }^{80}$ Este criterio ha sido recogido también en el caso Villagrán Morales y otros c. Guatemala ${ }^{81}$ y en el caso Godinez. Cruz c. Honduras. ${ }^{82}$

En el caso Mack Chang c. Venequela, la Corte IDH ha señalado que la abstención de proteger adecuadamente la escena del crimen, limpiar las uñas de la víctima y desechar el contenido de los raspados, no registrar ni conservar las huellas dactilares, no tomar muestra de sangre a la víctima, no someter a examen las ropas de la víctima, no fotografiar de forma completa las heridas de la víctima, etc., constituyen la omisión de

77 Kuznetsov vs. Ucrania, Application No. 39042/97, Sentencia (Merits and Just Satisfaction), 29 de abril de 2003, par. 106.

78 Tepe vs. Turquí, Application No. 27244/95, Sentencia (Merits and Just Satisfaction), 9 de mayo de 2003, pars. 181-182.

79 El Zeidy, The principle of complementarity..., 2008, 175-ss.

80 Velásquez Rodríguez c. Honduras, 29 de julio de 1988, Serie C. No 4, párr. 177.

81 Villagran Morales y otros c. Guatemala, 19 de noviembre de 1999, Serie C, No 63 (1999), par. 226.

82 Godinez Cruz c. Honduras, Sentencia, 20 de enero de 1989, Serie C. No 5 (1989), pars. 189-190. 
elementos fundamentales para el buen curso de la investigación judicial, especialmente cuando se está al frente de un hecho que le ha costado la vida a una persona. ${ }^{83}$

\subsection{Inadecuada recolección, uso de la prueba y admisión de diligencias probatorias}

Si los investigadores reúnen evidencia insuficiente o irrelevante, esto podría indicar un propósito de proteger a la persona en cuestión. Si en estos casos se encuentra que hay evidencia esencial que las autoridades sabían que existía pero que no recopilaron, la indicación es fuerte. ${ }^{84}$ Por cierto, lo anterior se entiende sin perjuicio de la responsabilidad penal "doméstica" de los investigadores institucionales respecto de algunas hipótesis delictivas contra la administración de justicia.

Este criterio también es recogido por la Oficina de la Fiscalía de la CPI, señalando que es un indicador de falta de autenticidad la incongruencia entre las decisiones frente a la prueba presentada. De igual forma pasar por alto pruebas o asignarles un peso insuficiente.

\subsection{Inadecuada acusación}

Otra forma de proteger al acusado es emitir una acusación inadecuada. En cuanto a la descripción objetiva, la acusación podría, por ejemplo, referirse a menos asesinatos que los realmente cometidos. De igual forma, la acusación podría no mencionar hechos importantes. Una descripción inadecuada del papel del autor en el crimen también podría ser relevante. La conducta podría describirse como la falta de control negligente de los subordinados, mientras que en realidad la persona ordenó la comisión de los delitos. Otra circunstancia fáctica esencial para los crímenes internacionales que podría no mencionarse es el contexto en el que se cometieron dichos ilícitos (conflicto armado, ataque generalizado, etc).

\footnotetext{
83 Myrna Mack Chang c. Guatemala, Sentencia (Fondo, Reparaciones y Costas) de 25 de noviembre de 2003. Serie C, No 101 (2003), párrs. 166 y 167; Stigen, The relationship between the international criminal court..., 2008: 275; El Zeidy, The principle of complementarity..., 2008, 166.

84 Stigen, The relationship between the international criminal court..., 2008, 281.
} 
También podría abarcar esta situación el hecho de que, a nivel doméstico, se enjuicie un crimen de competencia de la CPI como delito ordinario, a pesar de la existencia de una legislación interna adecuada. Esta situación puede equivaler a blindaje.

Este criterio también es recogido por la Oficina de la Fiscalía de la CPI, quien señala expresamente que es un indicador de falta de autenticidad las insuficiencias manifiestas en la formulación de los cargos y los modos de responsabilidad en relación con la gravedad de la conducta alegada. Asimismo, imprecisiones graves en el rol atribuido al acusado.

\subsection{Juicio irregular}

Si los funcionarios involucrados proceden de una manera que se desvía de los estándares internacionalmente reconocidos en favor del sospechoso, esto podría indicar un propósito de protección. Si no se sigue el iter procesal o se excluye prueba indebidamente, o si el juez no permite que los testigos de la acusación hagan declaraciones completas o permite que sean intimidados, estas son indicaciones relevantes en favor de la falta de autenticidad. ${ }^{85}$

Este criterio también es recogido por la Oficina de la Fiscalía de la CPI, quien consigna expresamente que son un indicador de "blindaje" las desviaciones de las prácticas y procedimientos establecidos. En el mismo sentido, la doctrina lo ha reconocido como indicio de falta de disposición. ${ }^{86}$

\subsection{Conclusiones judiciales equivocadas}

De lo que se trata este indicador es de que la decisión de adjudicación sea la manifestación de un vicio originado en la sentencia o en otras etapas procedimentales. Por ejemplo, que se arribe a una decisión equivocada a causa de una identificación errónea, un examen forense defectuoso, fallas de divulgación, pruebas fabricadas, declaraciones manipuladas o extraídas mediante coacción o decisiones indebidas de admisión o no de prueba. Este indicador permite resaltar una idea importante desde el

\footnotetext{
85 Stigen, The relationship between the international criminal court..., 2008, 282.

86 Ambos, 2009; Agirre et al, como se citó en Wolffhügel, "La falta de disposición...", 2009: 86-ss.
} 
plano procesal del enjuiciamiento doméstico: el dictado de una sentencia definitiva de un procedimiento judicial genuino presupone que en las fases o etapas anteriores no se hayan cometidos vicios manifiestos que se puedan expresar en la adjudicación. Ello, considerando especialmente la relación que exhiben las etapas del procediminto judicial con el dictado de la decisión adjudicatoria final. En el ámbito interno, la rectitud de la decisión final estará estrechamente vinculada a la satisfacción de las garantías del debido proceso en el enjuiciamiento que la antecede.

La doctrina también ha considerado indicadores de juicios no genuinos los altos niveles de absoluciones de determinados casos o condenas escuetas en casos de crímenes graves. ${ }^{87}$

\subsection{Otros indicadores}

Teniendo en cuenta que resulta muy complejo elaborar una lista completa y exhaustiva de todos los indicadores de falta de propósito de enjuciar al culpable, también se ha señalado como criterio las interferencias políticas, ${ }^{88}$ falta de trasparencia, inadecuada salida del juicio, inadecuada ejecución de la sentencia, lugar inapropiado del juicio, decisión inadecuada de no continuar con el procedimiento, asignación inapropiada del caso (falta de experiencia de sujetos institucionales), legislación inadecuada, omisión de pasos investigativos esenciales, utilización indebida de jueces o testigos anónimos, acción del Estado impulsada principalmente por las actuaciones de la fiscalía de la CPI, propósitos compartidos entre el Estado y el sospecho, etc. ${ }^{89}$

\section{La propuesta}

Todos los indicadores anteriores, principalmente a título orientativo, retratan un juzgamiento no "genuino" que, de haber finalizado, hubiese desembocado en una decisión adjudicatoria "manifiestamente injusta", en términos de Radbruch, intolerable para la comunidad internacional, que sustrae al sujeto de su responsabilidad internacional. Ello, por su parte, habilita la competencia de la CPI, a pesar de haberse ejercido

87 El Zeidy, The principle of complementarity..., 2008, 172.

88 Ellis, “The International Criminal Court...”, 2002: 239.

89 Stigen, The relationship between the international criminal court..., 2008, 282-ss. 
jurisdicción en el plano interno. ${ }^{90}$ Sin embargo, es posible que aún no se haya dictado la sentencia definitiva, en cuyo caso, igualmente la CPI podría llegar a estar limitada en su actuación por la prerrogativa del ne bis in idem procesal del artículo 20.3 del ER. Por otro lado, es posible que el procedimiento judicial, in itinere, sea genuino, pero no así la sentencia. En estos casos muestra su falencia una valoración individual o atomista de los indicadores.

La doctrina ha señalado que el propósito de sustraer a una persona de su responsabilidad individual debe ser evaluado requiriendo que la suma de las desviaciones individuales de la práctica estándar equivalga a una negligencia grave del Estado durante todo el proceso. ${ }^{91}$ Es decir, se debe ser cuidadoso al respecto porque finalmente se está evaluando cualitativamente la "justicia" del ejercicio jurisdiccional de los Estados.

¿Cómo realizar dicha conversión? Es decir, ¿cómo traducir la suma de indicadores individuales de enjuiciamientos no genuinos a una negligencia grave a lo largo de todo el proceso? En este sentido, se ha afirmado que debe haber una relación de causalidad entre propósito de blindaje y el o los pasos procesales inadecuados. Ello vendría denotado con la frase "resultado de" en el artículo 17 (1) (b) y "con el propósito de" en el factor (a), pero ello ha de ser tan claro que transforme en no genuino el procedimiento. ${ }^{92}$ De otro lado, también se ha dicho que la autenticidad de cualquier procedimiento judicial requiere el escrutinio de los procedimientos judiciales internos en relación con un caso específico, como un todo, desde el momento en que inicia hasta la etapa donde la valoración de la cuestión comienza. ${ }^{93}$

Una valoración global o de conjunto de los indicadores puede graficar que las etapas del procedimiento judicial presentan vicios manifiestos. De hecho, todos los indicadores muestran afectaciones en distintas etapas del procedimiento que culmina con la decisión adjudicatoria viciada. Si estoy en lo correcto al usar la nomenclatura procesal tradicional,

\footnotetext{
90 Gustav Radbruch, "Gesetzliches Unrecht und übergesetzliches Recht”. Süddeutsche Juristen-Zeitung, Jahrg. 1, Nr. 5 (August 1946):107; Gustav Radbruch, Introducción a la Filosofía del derecho. Traducc. Wenceslao Roces (México: Fondo de Cultura Económica, 1951), 44.

91 Dalpe, “On the Difficult Case...”, 2017: 77.

92 Stigen, The relationship between the international criminal court..., 2008, 282-ss.

93 El Zeidy, The principle of complementarity..., 2008, 166.
} 
se puede advertir que los indicadores afectan a cinco grandes etapas procedimentales: a) prejudicial, b) de investigación, c) de juzgamiento (discusión y prueba), d) de decisión y, e) de ejecución (tabla 1).

Tabla 1.

\begin{tabular}{|c|c|c|c|c|}
\hline $\begin{array}{c}\text { Etapa } \\
\text { prejudicial }\end{array}$ & $\begin{array}{c}\text { Etapa de } \\
\text { investigación }\end{array}$ & $\begin{array}{c}\text { Etapa de } \\
\text { juzgamiento }\end{array}$ & $\begin{array}{l}\text { Etapa de } \\
\text { decisión }\end{array}$ & $\begin{array}{l}\text { Etapa de } \\
\text { ejecución }\end{array}$ \\
\hline $\begin{array}{l}\text { - Renuencia a } \\
\text { cooperar con } \\
\text { la fiscalía de } \\
\text { la CPI. } \\
\text { - Limitación } \\
\text { al acceso de la } \\
\text { justicia. } \\
\text { - Inadecuada } \\
\text { asignación de } \\
\text { recursos. }\end{array}$ & $\begin{array}{l}\text { - Acceso y } \\
\text { seguridad de } \\
\text { los investiga- } \\
\text { dores. } \\
\text { - Intimidación } \\
\text { de los actores } \\
\text { del procedi- } \\
\text { miento. } \\
\text { - Omisión } \\
\text { de pasos } \\
\text { investigativos } \\
\text { esenciales. }\end{array}$ & $\begin{array}{l}\text { - Intimidación } \\
\text { de los actores del } \\
\text { procedimiento. } \\
\text { - Órganos judi- } \\
\text { ciales especiales. } \\
\text { - Minuciosidad } \\
\text { insuficiente en el } \\
\text { procedimiento. } \\
\text { - Inadecuada } \\
\text { recolección, uso } \\
\text { de la prueba } \\
\text { y admisión de } \\
\text { diligencias pro- } \\
\text { batorias. } \\
\text { - Inadecuada } \\
\text { acusación. } \\
\text { - Jueces o testi- } \\
\text { gos anónimos. } \\
\text { - Juicio irregular. }\end{array}$ & $\begin{array}{l}\text { - Intimidación } \\
\text { de los actores } \\
\text { del procedi- } \\
\text { miento. } \\
\text { - Órganos } \\
\text { judiciales } \\
\text { especiales. } \\
\text { - Minuciosidad } \\
\text { insuficiente } \\
\text { en el procedi- } \\
\text { miento. } \\
\text { - Jueces o testi- } \\
\text { gos anónimos. } \\
\text { - Juicio irre- } \\
\text { gular. } \\
\text { - Conclusiones } \\
\text { judiciales } \\
\text { Equivocadas. }\end{array}$ & $\begin{array}{l}\text { - Intimidación } \\
\text { de los actores } \\
\text { del procedi- } \\
\text { miento. } \\
\text { - Órganos } \\
\text { judiciales } \\
\text { especiales. } \\
\text { - Juicio irre- } \\
\text { gular. } \\
\text { - Conclusio- } \\
\text { nes judiciales } \\
\text { equivocadas. }\end{array}$ \\
\hline
\end{tabular}

Un procedimiento no genuino prototípico debiera ser capaz de mostrar indicadores en todas las etapas del procedimiento, porque se trata de valorar la "calidad" de justicia de un sistema judicial. Desde esta perspectiva, no solo es importante la presencia y prueba de un indicador, sino también la relación que este exhibe con la etapa de procedimiento y, en definitiva, con el enjuiciamiento de conjunto. Aquí se puede ver con nitidez la vertiente "holista" o de coherencia de la valoración probatoria antes anotada. Usando la misma analogía de Haack, nuestro crucigrama será el enjuiciamiento en su conjunto. De otro lado, aunque puede ser importante para valorar la autenticidad del procedimiento, creo que hay que tener cuidado con los indicadores que se han asignado a la etapa 
prejudicial, porque ello podría mostrar más bien una falta de capacidad del Estado para hacer responsable al acusado. ${ }^{94}$

La multiplicidad de indicadores, en otro sentido, puede sugerir el interrogante de si se puede considerar como no genuino un procedimiento donde concurra un solo indicador. En el ámbito probatorio, frente a la escasez de medios de prueba o dificultad de prueba (como podría ser la prueba de una circunstancia volitiva o de un estado) se suele recurrir al criterio de la corroboración. Es decir, la presencia de dos o más indicios — por regla general— que sean coherentes entre sí y que apoyen de manera conjunta lo que se intenta probar. Esta es la técnica que se usa, por ejemplo, en los casos de condenas penales basadas en el testimonio de la víctima (sección (32) (1)(b)). ${ }^{95}$

Frente a las dificultades probatorias, la tesis de la corroboración en el ámbito probatorio aconseja que, para dar lugar a la falta de disposición, los indicadores presentes sean dos o más que sean coherentes entre sí con la "intención o propósito de sustraer de su responsabilidad al acusado". Ello debiera ser el criterio general. De otro lado, los indicadores deberían estar presentes en dos o más etapas de procedimiento, pues de esta forma se elevan las probabilidades de que el procedimiento judicial en su conjunto no sea genuino. Sin embargo, ello también deberían tener en cuenta que no se debe transformar la cuestión en la verificación cuantitativa de indicadores solamente, pues lo relevante es ponderar la calidad del ejercicio jurisdiccional del Estado.

Pero, por otro lado, una valoración holista, de conjunto o global, a mi modo de ver, exige que dichos indicadores presenten tal importancia o magnitud que sean capaces de viciar gravemente al menos una de las etapas del procedimiento. Entre otras razones, porque la mayoría de los procedimientos penales modernos siguen un cierto orden consecutivo legal respecto de las etapas, de forma que viciada una se comunica el vicio o una consecuencia de mismo a las restantes, contaminando de esta forma todo el procedimiento y, por tanto, la vocación genuina del juzgamiento. Ello perfectamente puede alcanzarse por medio de la presencia de un solo indicador que, excepcionalmente, sea tan relevante y grave

\footnotetext{
94 Así, también, Stigen, The relationship between the international criminal court..., 2008, 268 ss.

95 Reino Unido, Criminal Justice and Public Order Act 1994, https://www.legislation. gov.uk/ukpga/1994/33/section/32
} 
que permita inferir que se ha viciado la etapa procedimental y, con ello, el procedimiento en su conjunto. La utilización de un solo indicador muy grave debe ser justificado, expresando claramente cómo este afecta a una etapa concreta del juicio y se proyecta al juicio en su conjunto.

Por el contrario, uno o más vicios que no alteren gravemente las etapas procedimentales no impiden que el juzgamiento pueda llegar a tener una vocación genuina, incluso una decisión justa o injusta que sea tolerable, porque no se trataría de un indicador epistémico que señale con claridad que el procedimiento es un medio que se utiliza para asegurar o sustraer al sujeto acusado de su responsabilidad por los crímenes cometidos. ${ }^{96}$

\section{Conclusión}

En el trabajo se ha ofrecido una visión probatoria de la cuestión de la falta de disposición en el caso que el Estado, al ejercer su jurisdicción, haya o esté sustrayendo a un individuo de su responsabilidad internacional —artículos 17. 2 (a) y 20.3 (a)—. Se aplica a ello la visión fundaherentista de Susan Haack, en virtud de la cual es necesario realizar una doble valoración de los indicios o indicadores a los que la doctrina y la jurisprudencia le reconocen valor para atribuir una falta de disposición por blindaje: una valoración holista y una atomista. El primero, vinculado a la gravedad del indicio para afectar gravemente alguna etapa del procedimiento jurisdiccional. El segundo, valorando la vocación epistémica de cada indicio.

Todos los indicadores afectan a distintas etapas del procedimiento (tabla 1) que culmina con la decisión adjudicatoria viciada. Un procedimiento no genuino prototípico debiera ser capaz de mostrar indicadores en todas las etapas del procedimiento. La multiplicidad de indicadores puede imprimir una imagen global de falta de autenticidad en el enjuiciamiento, siguiendo con ello la tesis de coherencia de Susan Haack. Frente a las dificultades probatorias, la tesis de la corroboración en el ámbito probatorio aconseja que, para dar lugar a la falta de

96 En un sentido bastante similar, pero desde otras perspectivas, Stigen señala que si el poder ejecutivo, separado del poder judicial, quiere proteger al autor, pero no puede influir en el poder judicial, y los procedimientos judiciales no se verán afectados y seguirán siendo genuinos. Stigen, The relationship between the international criminal court..., 2008, 263-ss. 
disposición, los indicadores presentes sean dos o más coherentes entre sí, que muestren adecuadamente la "intención o propósito de sustraer de su responsabilidad al acusado”. Sin embargo, no debe confiarse en exclusiva en una apreciación cuantitativa de los indicadores. El orden consecutivo legal presente en una buena parte de los procedimientos de la tradición románica continental y la gravedad que pueden llegar a presentar algunos indicios individualmente considerados, hacen posible que, en casos excepcionales, uno solo de ellos, con la debida justificación, pueda expresar la falta de disposición del Estado de someter a un individuo a su responsabilidad jurídica por actos cometidos.

La propuesta ha tenido por objeto hacer visible la perspectiva epistemológico-fáctica de la falta de disposición, como hipótesis excepcional de competencia de la CPI, la cual ha sido abordada por la doctrina - casi en exclusiva - desde un prisma normativo. En este sentido, ambas perspectivas son necesarias, complementarias y bajo ningún punto pueden considerarse excluyentes. Con todo lo anterior, también se quiso avanzar hacia un tratamiento sistemático y organizado de los indicadores, a partir de la tesis epistémica de Haack con la intención de contribuir respecto de un análisis más objetivo de la cuestión y no tan dependiente de las circunstancias de cada caso en concreto conocido por la CPI.

\section{Referencia}

Brighton, Claire. "Avoiding Unwillingness: Addressing the Political Pitfalls Inherent in the Complementarity Regime of the International Criminal Court". International Criminal Law Review 12(4) (2012): 629-664.

Cárdenas, Claudia. "La cooperación de los Estados con la Corte Penal Internacional a la luz del principio de complementariedad”. Revista de Derecho PUCV 1(34) (2010): 281-304.

Carter, Linda. "The Principle of Complementarity and the International Criminal Court: The Role of Ne Bis in Idem”. Santa Clara Journal of International Law 8(1) (2010): 165-198.

Chung, Chang-Ho. "The International Criminal Court 20 years after Rome-Achievements and deficits". En The International Criminal Court in Turbulent Times, editado por Gerhard Werle y Andreas Zimmermann, 9-22. The Hague: TMC Asser Press, 2019. https:// doi.org/10.1007/978-94-6265-303-0_2 
Dalpe, Vincent. "On the Difficult Case for a Functional Interpretation of the Unwillingness Criterion before the International Criminal Court". Journal of International Law and International Relations 13(2) (2017): 48-82.

Daza, Alfonso. Elprincipio de complementariedad en el derecho penal internacional. Bogotá: Universidad Católica de Colombia, 2015.

Ellis, Mark. "The International Criminal Court and Its Implication for Domestic Law and National Capacity Building". Florida Journal of International Law 15(2) (2002): 215-242.

El Zeidy, Mohamed. The principle of complementarity in International Criminal Law. Martinus Nijhoff publisher, 2008.

Ferrer, Jordi. La valoración racional de la prueba. Marcial Pons, 2007.

Finlay, Lorraine. "Does the International Criminal Court Protect against Double Jeopardy: An Analysis of Article 20 of the Rome Statute," UC Davis Journal of International Law \& Policy 15(2) (2009): 221-248.

Fuentes, Ximena. "El principio de complementariedad en la práctica de la Corte Penal Internacional". Estudios Internacionales 169, (2011): 119-140.

Haack, Susan. "Of Truth, in Science and in Law". Brooklyn Law Review 73(3) (2008): 985-1008. https://brooklynworks.brooklaw.edu/ $\mathrm{blr} / \mathrm{vol} 73 /$ iss $3 / 6$

Haack, Susan. “Defending Science-Within Reason”. Principia 2(3) (1999): 187-211.

Haack, Susan. "Federal Philosophy of Science: A Deconstruction and a Reconstruction". New York University Journal of Law and Liberty 5(2) (2010): 394-435.

Haack, Susan. "Proving Causation: The Holism of Warrant and the Atomism of Daubert". Journal of Health and Biomedical Law, (4) (2008): 253-258.

Holmes, John T. "Complementarity: National vs. The ICC". En The Rome statute of the International Criminal Court, editado por Antonio Cassese, Paola Gaeta y John R. W. D. Jones, 1:667-686. Oxford: Oxford University Press, 2002.

Kress, Claus. "The Procedural Law of the International Criminal Court in Outline: Anatomy of a Unique Compromise". Journal of International Criminal Justice 1(3) (2003): 603-617.

Llewellyn, Jennifer J. "A Comment on the Complementary Jurisdiction of the International Criminal Court: Adding Insult to Injury in Transitional Contexts". Dalhousie Law Journal 24(2) (2001): 192-217. 
McKeon, Patricia A. "An International Criminal Court: Balancing the Principle of Soveriegnty against the Demands for International Justice". St. John's Journal of Legal Commentary 12(2) (1997): 535-564. Nieva, Jordi. La cosa jurgada. Atelier, 2006.

Oficina de la Fiscalía, Corte Penal Internacional. Documento de politica general sobre exámenes preliminares. 2013. https://www.icc-cpi.int/ iccdocs/otp/OTP-Policy-Paper-on-Preliminary-ExaminationsNovember2013-SPA.pdf

Olásolo, Héctor. "Admisibilidad de situaciones y casos objeto de procesos de justicia de transición ante la corte penal internacional". En Ensayos de Derecho penal y procesal internacional, editado por Jessica Almqvist y Carlos Espósito, 255-289. Tirant lo Blanch, 2011.

Quesada, Carmen. "Levantando el velo de la lucha contra la impunidad: la corte penal internacional y sus intereses para actuar". En Actores internacionales contemporáneos, editado por Mónica Rocha, Carmen Quesada y Mario Iván Ureña, 225-256. Tirant lo Blanch, 2019.

Radbruch, Gustav. "Gesetzliches Unrecht und übergesetzliches Recht". Süddeutsche Juristen-Zeitung 1(5) (1946): 105-108.

Radbruch, Gustav. Introducción a la Filosofía del derecho. Traducido por Wenceslao Roces. México D. F.: Fondo de Cultura Económica, 1951.

Reino Unido. Criminal Justice and Public Order Act 1994. https://www. legislation.gov.uk/ukpga/1994/33/contents

Schabas, Williams \& El Zeidy, Mohamed. "Art.17”. En The Rome statute of The International Criminal Court, editado por Otto Triffterer y Kai Ambos, 782-832. Berlin: Beck/Hart, 2016.

Sternberg, Mark. "A Comparison of the Yugoslavian and Rwandan War Crimes Tribunals: Universal Jurisdiction and the Elementary Dictates of Humanity". Brooklyn Journal of International Law 22 (1996): 111.

Stigen, Jo. The relationship between the international criminal court and national jurisdictions. Martinus Nijhoff Publisher, 2008.

Taruffo, Michele. Simplemente la verdad: el juezy la construcción de los hechos. Traducido por Daniela Accantino Scagliotti. Marcial Pons, 2010.

Theofanis, Rosa. "The doctrine of res judicata in international criminal law". International Criminal Law Review, (3) (2003): 195-216.

Twining, William. Rethinking Evidence. Cambridge University Press, 2006. van der Vyver, Johan D. "Personal and Territorial Jurisdiction of the International Criminal Court". Emory International Law Review 14(1) (2000): 1-104. 
von Savigny, Friedrich. System des heutigen Römischen Rechts. Sechster Band. Bei Veit und Comp., 1847.

Werle, Gerhard \& Zimmermann, Andreas. "Introduction”. En The International Criminal Court in Turbulent Times, editado por Gerhard Werle y Andreas Zimmermann, 1-8. The Hague: TMC Asser Press, 2019. Wolffhügel, Christian. "La falta de disposición y de capacidad de los sistemas judiciales domésticos, en el marco del principio de complementariedad. A propósito del Estatuto de la Corte Penal Internacional". Civilizar. Ciencias Sociales y Humanas 9(17) (2009):79-88. https://www.redalyc.org/articulo.oa?id=100212301006

\section{Documentos}

(1995) Reporte del Comité ad hoc para la elabaración del Estatuto de la Corte Penal Internacional. 